TRANSACTIONS OF THE

AMERICAN MATHEMATICAL SOCIETY

Volume 355, Number 9 , Pages 3641-3650

S 0002-9947(03)03334-8

Article electronically published on May 15, 2003

\title{
ZERO ENTROPY, NON-INTEGRABLE GEODESIC FLOWS AND A NON-COMMUTATIVE ROTATION VECTOR
}

\author{
LEO T. BUTLER
}

\begin{abstract}
Let $\mathfrak{g}$ be a 2-step nilpotent Lie algebra; we say $\mathfrak{g}$ is non-integrable if, for a generic pair of points $p, p^{\prime} \in \mathfrak{g}^{*}$, the isotropy algebras do not commute: $\left[\mathfrak{g}_{p}, \mathfrak{g}_{p^{\prime}}\right] \neq 0$. Theorem: If $G$ is a simply-connected 2-step nilpotent Lie group, $\mathfrak{g}=\operatorname{Lie}(G)$ is non-integrable, $D<G$ is a cocompact subgroup, and $\mathbf{g}$ is a left-invariant Riemannian metric, then the geodesic flow of $\mathbf{g}$ on $T^{*}(D \backslash G)$ is neither Liouville nor non-commutatively integrable with $C^{0}$ first integrals. The proof uses a generalization of the rotation vector pioneered by Benardete and Mitchell.
\end{abstract}

\section{INTRODUCTION}

Let $\Sigma$ be a $C^{\infty}$ manifold, let $\mathbf{Z}(\Sigma)$ denote the set of $C^{\infty}$ Riemannian metrics on $\Sigma$ with zero topological entropy, and let $\mathbf{I}(\Sigma)$ denote those metrics with an integrable geodesic flow. Following Paternain [18, we can ask the questions:

Question A: Is the set of integrable geodesic flows $\mathbf{I}(\Sigma)$ nonempty? Is the set of zero-entropy geodesic flows $\mathbf{Z}(\Sigma)$ nonempty?

Question B: What is the topological structure of $\mathbf{I}(\Sigma)$, resp. $\mathbf{Z}(\Sigma)$ ?

The recent example in $[3$ by Bolsinov and Taumanov showed that $\mathbf{I}(\Sigma) \not Z \mathbf{Z}(\Sigma)$.

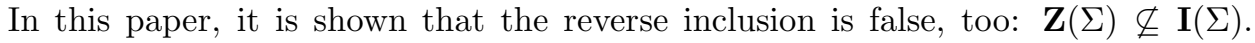
Thus, zero topological entropy is neither a necessary nor a sufficient condition for integrability.

There are well-known topological obstructions to the existence of a zero-entropy geodesic flow on a compact manifold: exponential word growth of the fundamental group and exponential growth of the Betti numbers of the loop space (over any field) [18. An interesting class of manifolds where neither of these two wellknown obstructions is effective is the class of nilmanifolds: quotients of nilpotent Lie groups. The fundamental groups of these manifolds are of polynomial word growth [1], and they are aspherical; so the loop space has trivial homology in all but the 0 -th group. This paper constructs 2 -step nilmanifolds on which all leftinvariant geodesic flows are non-integrable, but their entropy is zero. It is unclear if these manifolds admit any geodesic flows that are integrable. It seems likely that they do not.

Received by the editors May 13, 2002 and, in revised form, September 23, 2002.

2000 Mathematics Subject Classification. Primary 37J30, 37E45; Secondary 53D25.

Key words and phrases. Rotation vector, geodesic flows, entropy, nilmanifolds, nonintegrability. Research partially supported by a Natural Sciences and Engineering Research Council of Canada Postdoctoral Fellowship. Thanks to Gabriel Paternain, John Franks and Queen's University. 
There has been some work related to the theme of this paper. Eberlein, Lee and Park, and Mast (amongst others) have studied the question of whether a leftinvariant Riemannian metric $\mathbf{g}$ on the two-step nilpotent Lie group $G$ has a dense set of periodic points on $S(\Gamma \backslash G)$ for all lattice subgroups $\Gamma$ [10, [11, [15], [17. One way to understand this work is that the authors use the integrability of the geodesic flows in question to prove their results; the use of the formalism of the Euler-Lagrange equations on $T G$ rather than Hamilton's equations on $T^{*} G$ obscures this fact, however. In [4, [5], 6], explicit examples of integrable geodesic flows are constructed on a number of families of 2-step nilmanifolds including those studied in [10, 11], [15]. In [6] it is also shown that every left-invariant geodesic flow on every 2-step nilmanifold has zero topological entropy. The paper 7 constructs completely integrable, zero-entropy geodesic flows on an $n$-step filiform 1 nilmanifold for all $n$, thus proving that neither the step length nor the growth of the step length relative to the dimension of the group is an obstruction to the existence of integrable or zero-entropy geodesic flows. Finally, in $[8$, examples are constructed of $n$-step nilmanifolds, $n \geq 3$, with left-invariant metrics that have positive topological entropy.

The present paper constructs explicit examples of 2-step nilmanifolds whose leftinvariant geodesic flows are not integrable.

Let us formulate the results of this paper more precisely. Let $\mathfrak{g}$ be a Lie algebra and $p \in \mathfrak{g}^{*}$; denote by $\mathfrak{g}_{p}=\left\{x \in \mathfrak{g}: \operatorname{ad}_{x}^{*} p=0\right\}$. The point $p \in \mathfrak{g}^{*}$ is regular if $\operatorname{dim} \mathfrak{g}_{p}$ is minimal; a pair of points $p, p^{\prime} \in \mathfrak{g}^{*}$ is generic if $\operatorname{dim}\left[\mathfrak{g}_{p}, \mathfrak{g}_{p^{\prime}}\right]$ is minimal.

Definition 1.1. Let $\mathfrak{g}$ be a 2-step nilpotent Lie algebra; $\mathfrak{g}$ will be said to be nonintegrable if, for a dense set of generic pairs of points $p, p^{\prime} \in \mathfrak{g}^{*}$, we have $\left[\mathfrak{g}_{p}, \mathfrak{g}_{p^{\prime}}\right] \neq 0$.

Lemma 3.2 proves that Definition 1.1 is equivalent to the existence of a generic pair of points $p, p^{\prime} \in \mathfrak{g}^{*}$ such that $\left[\mathfrak{g}_{p}, \mathfrak{g}_{p^{\prime}}\right] \neq 0$. A notion related to non-integrability is almost non-singularity: $\mathfrak{g}$ is almost non-singular if there exists a regular point $p \in \mathfrak{g}^{*}$ such that the 2 -form $d p$ defined for all $x, y \in \mathfrak{g}$ by $d p(x, y)=-\langle p,[x, y]\rangle$ induces a symplectic form on $\mathfrak{g} /[\mathfrak{g}, \mathfrak{g}][15]$. If $\mathfrak{g}$ is almost non-singular, then for all regular points $p \in \mathfrak{g}^{*}, \mathfrak{g}_{p}=Z(\mathfrak{g})$. Non-integrable 2-step nilpotent Lie algebras cannot be almost non-singular 2-step nilpotent Lie algebras. There are, however, 2step nilpotent Lie algebras that are neither almost non-singular nor non-integrable. The example of $\mathfrak{g}=\operatorname{span}\left\{x, y_{1}, \ldots, y_{n}, z_{1}, \ldots, z_{n}\right\}$ with the relations $\left[x, y_{i}\right]=z_{i}$ is studied in [4, [5].

Here are two elementary constructions of a non-integrable 2-step nilpotent Lie algebra. Let $\mathfrak{h}$ be a real, semi-simple Lie algebra and let $\mathfrak{g}=\mathfrak{h} \oplus \mathfrak{h}$ be the vector space direct sum of 2 copies of $\mathfrak{h}$, with Lie bracket defined by $\left[x \oplus y, x^{\prime} \oplus y^{\prime}\right]=0 \oplus\left[x, x^{\prime}\right]$. The example to keep in mind is $\mathfrak{h}=s o(3)$. A second example is this: let $V$ be a real, odd-dimensional vector space and let $\Lambda^{2}(V)$ be the vector space of alternating forms on $V^{*}$ and let $\mathfrak{g}=V \oplus \Lambda^{2}(V)$ with Lie bracket $\left[x \oplus y, x^{\prime} \oplus y^{\prime}\right]=0 \oplus x \wedge x^{\prime}$. The lowest dimension for a non-integrable 2-step nilpotent Lie algebra is five: let $\mathfrak{g}_{6}=\mathbf{R}^{3} \oplus \Lambda^{2}\left(\mathbf{R}^{3}\right)$ and let $Z \subset Z\left(\mathfrak{g}_{6}\right)$ be a one-dimensional subalgebra; then $\mathfrak{g}_{5}:=\mathfrak{g}_{6} / Z$ is also non-integrable. One can verify that there are no further examples in dimensions 3 or 4 .

\footnotetext{
${ }^{1}$ The step length of a nilpotent Lie algebra of dimension $d \geq 2$ is bounded by $d-1$; it is filiform if it attains this bound.
} 
Definition 1.2 (cf. [14]). Let $\phi_{t}: M \rightarrow M$ be a $C^{0}$ flow. It is integrable if there exists an open dense subset $L \subset M$ such that $L$ is a $C^{0}$ torus fibre bundle, and the fibres of $L$ are $\phi_{t}$-invariant.

The hypothesis that $L$ is a $C^{0}$ torus fibre bundle means that there is a $C^{0}$ manifold $B$ and $L$ is a locally trivial fibre bundle over $B$ with fibres $\simeq \mathbf{T}^{k}$. The most common way to obtain integrable systems is via the Liouville-Arnol'd-Nehorošev theorem for Hamiltonian systems.

Theorem 1.3 (Main Theorem). Let $G$ be a simply-connected 2-step nilpotent Lie group, $\mathfrak{g}=\operatorname{Lie}(G)$ be non-integrable, $D<G$ be a cocompact subgroup, and $\mathbf{g}$ be a left-invariant Riemannian metric. Let $\Sigma=D \backslash G$. Then the geodesic flow of $\mathbf{g}$ on $M=T^{*} \Sigma$ is not integrable in the sense of Definition 1.2. In particular, it is neither Liouville nor non-commutatively integrable with $C^{0}$ first integrals.

Let us observe that not all nilpotent Lie groups have discrete cocompact subgroups; the existence of such a subgroup is known to be equivalent to the existence of a $\mathbf{Q}$-structure on $\mathfrak{g}$ [16].

1.1. Outline. The proof of Proposition 1.3 is surprisingly straightforward and is related to a generalization of a rotation vector to non-commutative groups. Schwartzman [20. (see also [19]) introduced the notion of an asymptotic homology class in $H_{1}(M ; \mathbf{R})$ for any curve $c: \mathbf{R} \rightarrow M$. Recall that $H_{1}(M ; \mathbf{R})=\pi /[\pi, \pi] \otimes \mathbf{R}$, where $\pi=\pi_{1}(M)$. This asymptotic homology class was generalized by Benardete and Mitchell [2] - who were building on the work of Chen [9] - to an asymptotic homotopy class of the curve $c$. Let us sketch the definition of this homotopy class. This principally requires an explanation of where it lives. Assume that $\pi$ is a finitely-generated group. Let $\delta_{0}:=\pi$ and $\delta_{n+1}:=\left[\pi, \delta_{n}\right]$. In general, $\pi / \delta_{n}$ has torsion, but the radical of $\delta_{n}, \Delta_{n}=\sqrt{\delta_{n}}$, is a normal subgroup of $\pi$ such that $\pi^{n}:=\pi / \Delta_{n}$ is torsion-free. It is known that $\pi^{n}$ is finitely-generated, $n$-step nilpotent and torsion-free. By a theorem of Mal'cev 16, there is a connected, simply-connected $n$-step nilpotent Lie group $N_{n}$ such that $\pi^{n}$ is a discrete, cocompact subgroup of $N_{n}$. The group $N_{n}$ is unique up to isomorphism. Note that $\pi^{1} \simeq H_{1}(M ; \mathbf{Z}) /$ Tor $H_{1}(M ; \mathbf{Z})$ and that $N_{1} \simeq H_{1}(M ; \mathbf{R})$. This construction, called the Mal'cev completion of $\pi$, is a generalization of the standard tensor product. The asymptotic homotopy class defined by Benardete and Mitchell lives in the connected, simply-connected 2-step nilpotent Lie group $N_{2}$. Let us note that the construction of the groups $N_{n}$ and $\Delta_{n}$ is "pointed" because $\pi=\pi(M ; q)$ is pointed; so we should use the notation $\left\{N_{n, q}, \Delta_{n, q}\right\}$. A particularly nice feature of Benardete and Mitchell's paper [2] is that they embed the family of groupswith-lattice-subgroups $\left\{N_{n, q}, \Delta_{n, q}\right\}_{q \in M}$ in a connected, simply-connected $n$-step nilpotent Lie group $N_{n}$ with lattice $\Delta_{n}$ and then show that there are isomorphisms $\phi_{q}: N_{n, q} \rightarrow N_{n}$ (which satisfy $\phi_{q}\left(\Delta_{n, q}\right)=\Delta_{n}$ ) such that $\phi_{q^{\prime}} \circ \phi_{q}^{-1}$ is an inner automorphism of $N_{n}$ for all $q, q^{\prime} \in M$. Of course, there are no canonical choices for the $\phi_{q}$.

The precise definition of the asymptotic homotopy class in $N_{2}$ requires some work. There is no ergodic theorem for these cocycles; so it is unknown if the asymptotic homotopy class exists for almost all initial conditions. Because the interest is really in understanding the asymptotic homotopy classes for a flow, we have elected to follow Fried [12] and define a related notion: projective homotopy classes. We show that it is possible to put enough algebraic structure on these 
asymptotic homotopy classes in order to derive an effective necessary condition for integrability (see Lemma 2.7). Combined with a few calculations in section 3, this necessary condition gives a proof of Proposition 1.3 .

\section{Free projective asymptotic homotopy Classes}

Let us continue with the notation in the outline. Let $N$ be an $n$-step nilpotent Lie group and define $\mathbb{P} N$ to be the set of all one-parameter subgroups of $N$. Recall that if $N$ is a connected, simply-connected nilpotent Lie group, then, by identifying $N \simeq \operatorname{Lie}(N)$ via the exponential map, we can identify $\mathbb{P} N$ with $\mathbb{P} \mathfrak{n}=\mathfrak{n} / \mathbf{R}$, where $\mathfrak{n}:=\operatorname{Lie}(N)$. $\mathbb{P} \mathfrak{n}$ is the set of all subspaces of $\mathfrak{n}$ with dimension at most 1 . We can equip $\mathbb{P} \mathfrak{n}$ with the topology induced by the projection map $\pi(x)=\mathbf{R} x$, which makes it into a compact, connected non-Hausdorff topological space. We will also denote the coset $\mathbf{R} x$ by $\bar{x}$. The set $\{\overline{0}\} \subset \mathbb{P n}$ will be said to be trivial. Let us state the following:

Lemma 2.1. Let $L$ be a real Lie algebra, $\mathbb{P} L=L / \mathbf{R}$. Then

1) if $\phi: L \rightarrow L$ is a linear transformation, there is a continuous map $\bar{\phi}: \mathbb{P} L \rightarrow \mathbb{P} L$ such that $\pi \circ \phi=\bar{\phi} \circ \pi$;

2) for all $\bar{x} \in \mathbb{P} L$ there is a continuous map $\overline{\operatorname{ad}_{\bar{x}}}: \mathbb{P} L \rightarrow \mathbb{P} L$ such that for all $x \in \pi^{-1}(\bar{x}): \pi \circ \operatorname{ad}_{x}=\overline{\operatorname{ad}_{\bar{x}}} \circ \pi$.

The proof of Lemma 2.1 is straightforward. We note that (1) implies that the adjoint representation of $N$ on $\mathfrak{n}$ descends to an action on $\mathbb{P} \mathfrak{n}$, while (2) shows that, if $L=\mathfrak{n}$ is nilpotent, then we can introduce a grading on $\mathbb{P} \mathfrak{n}$ by saying that $\bar{x} \in \mathbb{P}^{k}$

iff $\overline{\operatorname{ad}}_{\bar{x}}^{k}=\overline{0}$ and $\overline{\operatorname{ad}}_{\bar{x}}^{k-1} \neq \overline{0}$. This grading is inherited from the grading of $\mathfrak{n}$ by its derived subalgebras. Part (2) also implies that the projective bracket $[\bar{x}, \bar{y}]$ is well-defined; it is also well-defined to say that $\bar{x}$ and $\bar{y}$ commute. This is equivalent to $[x, y]=0$ for all $x \in \bar{x}$ and $y \in \bar{y}$, which is equivalent to $[\exp (t x), \exp (s y)]=1$ for all $s$ and $t$.

We are interested in the case where $N=N_{n}$ is the $n$-th part of the Mal'cev completion of $\pi=\pi_{1}(M ; q)$. Let $\Pi: N \rightarrow \mathbb{P n}$ be defined by $\Pi=\pi \circ \log$. We want to define:

Definition 2.2. Let $c:[0, \infty) \rightarrow N$ be a continuous curve, $c(0)=1$. Let $C_{t}:=$ $\{\Pi(c(s)): s \geq t\}$ and define

$$
[c]=\bigcap_{t>0} \overline{C_{t}} \subset \mathbb{P n}
$$

to be the projective homotopy class of $c$. If $c^{\prime}(t):=g c(t)$ for some $g \in N$, then $\left[c^{\prime}\right]:=\overline{\operatorname{Ad}_{g}}[c]$.

Note that $\overline{0}$ lies in any nonempty closed subset of $\mathbb{P} \mathfrak{n}$. So $[c]$ always contains the trivial subset $\{\overline{0}\}$. For this reason, let us define the following set mapping $\hat{\pi}(V):=\pi(V) \cup\{\overline{0}\}$ for any $V \subseteq \mathfrak{n}$.

We can compute $[c]$ as follows: put some norm |.| on $\mathfrak{n}$, and let

$$
d(t)=\exp \left(|\log c(t)|^{-1} \log c(t)\right)
$$

if $c(t) \neq i d$ and 0 otherwise. Then $[c]=[d]$. This shows that $\overline{0} \neq \bar{x} \in[c]$ iff there is a sequence $x_{k} \in \mathfrak{n}$ of norm 1 such that $d\left(t_{k}\right)=x_{k}$ and $x_{k} \rightarrow x$. Thus, the projective homotopy class is a generalization of the homology directions (a projective rotation 
vector) of Fried. The following two lemmata might be interpreted to say that Definition 2.2 is "correct".

Lemma 2.3. Let $x \in \mathfrak{n}, g \in N, c(t):=e^{t x}, c^{\prime}(t):=g c(t)$ and $c^{\prime \prime}(t):=g c(t) g^{-1}$. Then $[c]=\hat{\pi}(x)$ and $\left[c^{\prime}\right]=\left[c^{\prime \prime}\right]=\hat{\pi}\left(\operatorname{Ad}_{g} x\right)$.

Proof. The map $\Pi: N \rightarrow \mathbb{P} \mathfrak{n}$ satisfies $\Pi\left(e^{t x}\right)=\pi\left(\log \left(e^{t x}\right)\right)=\pi(x)$ for $t \neq 0$. Thus $C_{t}=\{\pi(s x): s \geq t\}=\pi(x)$ and so $\overline{C_{t}}=\hat{\pi}(x)$. The remaining claims are clear.

Given a continuous map $\gamma: \mathbf{T}^{1} \rightarrow M$ we can extend $\gamma$ to a map $\mathbf{R} \rightarrow M$. Let this map be denoted by $\hat{\gamma}$.

Let $N$ be a connected, simply-connected nilpotent Lie group, $D<N$ a discrete, cocompact subgroup and let $\Sigma=D \backslash N$. For $\sigma=D g$ there is a (non-canonical, nonunique) identification of $\pi_{1}(\Sigma ; \sigma)$ with $\pi_{1}(\Sigma ; D e)=D$ by the map $\gamma(t) \rightarrow \gamma(t) g^{-1}$.

Lemma 2.4. Assume that $D<N$ is a discrete, cocompact subgroup and let $\Sigma=$ $D \backslash N$. Let $\gamma \in \pi_{1}(\Sigma ; \sigma)$ be a nontrivial homotopy class, $\sigma=D g$ and let $\Gamma: \mathbf{R} \rightarrow N$ be a lift of $\hat{\gamma}$ based at $g$. If $\gamma$ is homotopic to the loop $t \rightarrow D g e^{t x}$, then $[\Gamma]=$ $\hat{\pi}\left(\operatorname{Ad}_{g} x\right)$.

Proof. Assume that $\sigma=D e, \Gamma(0)=e$, and let $d=\Gamma(1) \in D$. By hypothesis $d \neq e$. Then, there is a nonzero $x \in \mathfrak{n}$ such that $d=e^{x}$. Let $c(t)=e^{t x}$; so that $c(n)=d^{n}$ for all $n \in \mathbf{Z}$. The map $q(t)=\Gamma(t) c(t)^{-1}$ is 1-periodic and continuous; so $\operatorname{im} q$ is a compact subset of $N$. Since $\Gamma(t)=q(t) c(t)$, we can write that $\log \Gamma(t)=t x+p(t)$. The set $\operatorname{im} p$ is also compact; so $\log \Gamma(t) /|\log \Gamma(t)|=x /|x|+O\left(t^{-1}\right)$ is well-defined for all $t$ sufficiently large. Then $[\Gamma]=\hat{\pi}(x)$.

In the general case, we non-canonically identify $\pi_{1}(\Sigma ; D g)$ with $\pi_{1}(\Sigma ; D e)$ via the map $\gamma(t) \rightarrow \gamma(t) g^{-1}=: \tilde{\gamma}(t)$. From the arguments of the previous paragraph we can assume, without loss of generality, that $\gamma(t)=D g e^{t x}$. Then $\tilde{\gamma}(t)=D e^{t \operatorname{Ad}_{g} x}$. Apply Lemma 2.3 and the results of the previous paragraph to obtain the conclusion.

The $\pi_{1}$ de Rham theorem of Benardete and Mitchell allows one to introduce a second notion, which is more suitable for flows. Let $\phi_{t}: M \rightarrow M$ be a $C^{1}$ semi-flow, and let $q \in M$ and $n \geq 1$ be fixed. The Mal'cev completion of $\pi_{1}(M ; q)$ induces a map $f_{q, n}: C^{0}((\mathbf{R}, 0),(M, q)) \rightarrow N_{n}$. Let us denote a projective homotopy class in $\mathbb{P} \mathfrak{n}$ by $[.]_{n}$. We define the projective homotopy class of $G(t)=\phi_{t}(q)$ to be $\left[f_{q, n} \circ G\right]_{n}$ and denote this by $[q]_{n, \phi}$. In the sequel, we will drop the subscript $n, \phi$ when this is understood. Let us also introduce a convenient notation: if $V \subset \mathfrak{n}$ (resp. $W \subset \mathbb{P} \mathfrak{n}$ ), then $\mathcal{O}(V):=\left\{\operatorname{Ad}_{g} v: g \in N, v \in V\right\}\left(\right.$ resp. $\left.\overline{\mathcal{O}}(W)=\left\{\overline{\operatorname{Ad}_{g}} w: g \in N, w \in W\right\}\right)$. It is clear that $\overline{\mathcal{O}}(\pi(V))=\pi(\mathcal{O}(V))$.

Definition 2.5. The free projective asymptotic homotopy class of $q$ is the set

$$
\mathcal{F}_{n, \phi}(q):=\overline{\mathcal{O}}\left([q]_{n, \phi}\right) .
$$

By Theorem 3.1 of [2] and Lemma 2.1, if $p=\phi_{t_{0}}(q)$, then $[q]=\overline{\operatorname{Ad}_{g}}[p]$ for some $g \in N_{n}$. Thus, the free projective asymptotic homotopy class is an invariant of the orbit $\left\{\phi_{t}(q)\right\}_{t \in \mathbf{R}}$. In addition, although the construction of Theorem 3.1 in [2] depends on the smoothness of the manifold $M$, the free projective homotopy classes are an invariant of the $C^{0}$ conjugacy class of the semi-flow $\phi_{t}$. In certain cases, it is possible to see this directly: when $M$ is a vector bundle over a nilmanifold, the free projective asymptotic homotopy classes can be defined directly from Definition 2.2 without recourse to Theorem 3.1 of [2]. Note that Lemma[2.4]implies that Definition 
2.5 is also "correct": if $\phi_{t}(q)=q e^{t x}$, then the projective asymptotic homotopy class of $c_{q}(t)=q e^{t x}$ is $\left[c_{q}\right]=\hat{\pi}\left(\operatorname{Ad}_{q} x\right)$, while the free projective asymptotic homotopy class is $\mathcal{F}_{\phi}(q)=\overline{\mathcal{O}}\left(\left[c_{q}\right]\right)=\bigcup_{g \in N}\left[c_{g}\right]$.

Let us now explore the relationship of $\mathcal{F}_{n, \phi}(q)$ with integrability.

Definition 2.6. Let $U, V \subset \mathbb{P n}$. We say that $U, V$ weakly commute if there exist nontrivial $\bar{x} \in U, \bar{y} \in V$ such that $[\bar{x}, \bar{y}]=\overline{0}$.

Lemma 2.7. Let $M$ be a compact manifold and $\phi_{t}: M \rightarrow M$ be a $C^{0}$ semi-flow. Assume that there is a path-connected $\phi_{t}$-invariant set $U \subset M$ such that $\pi_{1}(U)$ is abelian. Then for all $u, u^{\prime} \in U$ and all $n \geq 1$, either $\mathcal{F}_{n, \phi}(u)$ and $\mathcal{F}_{n, \phi}\left(u^{\prime}\right)$ weakly commute or at least one of $\mathcal{F}_{n, \phi}(u), \mathcal{F}_{n, \phi}\left(u^{\prime}\right)$ is trivial.

Proof. Let $i: U \rightarrow M$ denote the inclusion map; $i$ maps $\pi_{1}(U ; u)$ into an abelian subgroup of $\pi_{1}(M ; i(u))$ for all $u \in U$. By the naturality of the Mal'cev completion of $\pi_{1}(M)$, for each $n \geq 1, i_{*} \pi_{1}(U ; u)$ gets mapped to a discrete torsion-free abelian subgroup $\mathbf{A}_{n}$ of $\Delta_{n}$, and so there is an abelian subgroup $A_{n} \subseteq N_{n}$ such that $A_{n}=\exp \left(\operatorname{span} \log \left(\mathbf{A}_{n}\right)\right)$. The positive-invariance of $U$ implies that $\mathcal{F}_{n, \phi}(u)$ and $\mathcal{F}_{n, \phi}\left(u^{\prime}\right)$ are subsets of $A_{n}$. Therefore, if there are nontrivial elements in both $\mathcal{F}_{n, \phi}(u)$ and $\mathcal{F}_{n, \phi}\left(u^{\prime}\right)$, then there are nontrivial elements in each set that commute; so the sets weakly commute. Otherwise, at least one of the sets is trivial.

Corollary 2.8. Let $\phi_{t}$ be a $C^{0}$ semi-flow. Assume that there is a $C^{0}$ embedding $i$ of $U \simeq \mathbf{T}^{k} \times \mathbf{D}^{l}$ into $M$, such that $i(U)$ is $\phi_{t}$-invariant. Assume that there is a $C^{0}$ map $\omega: \mathbf{D}^{l} \rightarrow \mathbf{R}^{k}$ such that for all $\theta \in \mathbf{T}^{k}, I \in \mathbf{D}^{l}$ we have $i^{-1} \phi_{t} i(\theta, I)=(\theta+t \omega(I), I)$. Then for all $u, u^{\prime} \in U$ and all $n \geq 1$, either the sets $\mathcal{F}_{n, \phi}(i(u))$ and $\mathcal{F}_{n, \phi}\left(i\left(u^{\prime}\right)\right)$ weakly commute or at least one is trivial.

\section{TWO-STEP NILPOTENT LIE GROUPS}

Let $\mathfrak{g}$ be a 2 -step nilpotent Lie algebra with center $\mathfrak{z}=Z(\mathfrak{g})$, so that $[\mathfrak{g}, \mathfrak{g}] \subset Z(\mathfrak{g})$, let $\langle$,$\rangle be an inner product on \mathfrak{g}$, and let

$$
\mathfrak{g}=\mathfrak{h} \oplus \mathfrak{z}
$$

be an $\langle$,$\rangle -orthogonal decomposition of \mathfrak{g}$. The Lie bracket on $\mathfrak{g}$ is written as $\left[x+y, x^{\prime}+y^{\prime}\right]=\left[x, x^{\prime}\right]$ for all $x, x^{\prime} \in \mathfrak{h}$ and $y, y^{\prime} \in \mathfrak{z}$, and so the commutator defines a skew-symmetric, bilinear form $\omega: \mathfrak{h} \times \mathfrak{h} \rightarrow \mathfrak{z}$ by $\omega\left(x, x^{\prime}\right)=\left[x, x^{\prime}\right]$.

The Lie algebra $\mathfrak{g}$ can also be given the structure of a Lie group $(G, *)$ by $X * Y:=X+Y+\frac{1}{2}[X, Y]$, so that $\mathfrak{g}=\operatorname{Lie}(G)$ and the exponential map is the identity. In the sequel, elements in $G$ will often be viewed as elements in $\mathfrak{g}$ under the inverse (logarithm) map - which is the identity map in these coordinates. If $D$ is a discrete, cocompact subgroup of $G$, then there exists a generating set $X_{1}, \ldots, X_{p}, Y_{1}, \ldots, Y_{q}$ where $Y_{1}, \ldots, Y_{q}$ generate $Z(D)$ and the cosets $X_{1}+Z(D), \ldots, X_{p}+Z(D)$ generate $D / Z(D)$ and $p=\operatorname{dim} \mathfrak{h}, q=\operatorname{dim} \mathfrak{z}$ [16]. The generating set therefore determines a basis of $\mathfrak{g}$ and an inner product $\langle,\rangle^{\prime}$ relative to which it is an orthonormal basis. It may be supposed then that $\langle\rangle=,\langle,\rangle^{\prime}$, $\mathfrak{h}=\operatorname{span}_{\mathbf{R}}\left\{X_{1}, \ldots, X_{p}\right\}$ and $\mathfrak{z}=\operatorname{span}_{\mathbf{R}}\left\{Y_{1}, \ldots, Y_{q}\right\}$.

Lemma 3.1. Let $D \leq G$ be a discrete, cocompact subgroup and let (, ) be an inner product on $\mathfrak{g}$. Then there exists an automorphism $f: G \rightarrow G$ and a subgroup $D^{\prime}=f^{-1}(D)$ with generators $X_{1}, \ldots, X_{p}, Y_{1}, \ldots, Y_{q}$ such that $\left(X_{i}, Y_{j}\right)=0$. In 
addition, if $\mathbf{g}$ is the left-invariant metric on $G$ determined by $($,$) , then \left(D^{\prime} \backslash G, f^{*} \mathbf{g}\right)$ is isometric to $(D \backslash G, \mathbf{g})$.

Proof. Let $\mathfrak{g}=\mathfrak{h} \oplus \mathfrak{z}$ be the $\langle$,$\rangle -orthogonal decomposition of \mathfrak{g}$. Let $a(x)$ be the $($,$) -orthogonal projection of x \in \mathfrak{h}$ onto $\mathfrak{z}$. The map $F: x+y \rightarrow x-a(x)+y$ for all $x \in \mathfrak{h}$ and $y \in \mathfrak{z}$ is an automorphism of $\mathfrak{g}$. Let $f=\exp \circ F \circ \log$ be the map induced by $F$ on $G$. Then $f$ is an automorphism and by construction $F(\mathfrak{h})$ is $($,$) -orthogonal$ to $\mathfrak{z}$.

Lemma 3.1 is proven in 13 for Heisenberg groups. The importance of this lemma is that, by fixing a discrete, cocompact subgroup $D$ with a fixed generating set, attention can be confined to those metrics that are block diagonal relative to this fixed basis of $\mathfrak{g}$. Here and henceforth, $\langle$,$\rangle will be a fixed inner product on \mathfrak{g}$ relative to which $\mathfrak{g}=\mathfrak{h} \oplus \mathfrak{z}, D$ will be a discrete, cocompact subgroup of $G$ with $\langle$,$\rangle -orthonormal generating set X_{1}, \ldots, X_{p}, Y_{1}, \ldots, Y_{q}$ and $($,$) will be a second inner$ product that is block diagonal: for all $X, X^{\prime} \in \mathfrak{h}$ and $Y, Y^{\prime} \in \mathfrak{z}$,

$$
\left(X+Y, X^{\prime}+Y^{\prime}\right)=\langle X, \mathcal{A} X\rangle+\left\langle Y, \mathcal{B} Y^{\prime}\right\rangle
$$

where $\mathcal{A}_{i j}=\left(X_{i}, X_{j}\right)$ and $\mathcal{B}_{k l}=\left(Y_{k}, Y_{l}\right)$. The metric $\mathrm{g}$ on $G$ will be the leftinvariant metric determined by $($, ) or, equivalently, the pair $\mathcal{A}, \mathcal{B}$.

Finally, let $\mathfrak{g}$ be a real, nonabelian Lie algebra and let $r=\min _{p \in \mathfrak{g}^{*}} \operatorname{dim} \mathfrak{g}_{p} ; r$ is commonly called the index of $\mathfrak{g}$. It is well-known that the set of $p$ where $\operatorname{dim} \mathfrak{g}_{p}>r$ is a nontrivial algebraic set; let $\mathfrak{g}_{r}^{*}$ denote the complement of this set. Let $G_{r}$ denote the Grassmannian of $r$-dimensional subspaces of $\mathfrak{g}$, and let $\mathbf{G}_{r}=G_{r} \times \mathfrak{g}_{r}^{*}$ denote the trivial $G_{r}$ bundle over $\mathfrak{g}_{r}^{*}$. The map $p \rightarrow \mathfrak{g}_{p}$ restricted to $\mathfrak{g}_{r}^{*}$ is a section of $\mathbf{G}_{r}$. Since $x \in \mathfrak{g}_{p}$ iff $\operatorname{ad}_{x}^{*} p=0$ iff $\sum_{\alpha, \beta=1}^{n} c_{\beta, i}^{\alpha} p_{\alpha} x^{\beta}=0$ for $i=1, \ldots, n$ (where $c_{\beta, i}^{\alpha}$ are the structure constants of $\mathfrak{g}$ ) iff $x$ satisfies a system of linear equations with coefficients linear functions of $p$, so it follows that the section $p \rightarrow \mathfrak{g}_{p}$ is algebraic in $p$. Thus, the map $f: \mathfrak{g}_{r}^{*} \rightarrow \mathfrak{g}$ defined by $p \rightarrow\left[\mathfrak{g}_{p}, \mathfrak{g}_{p}\right]$ is algebraic and so the subset $\{f \neq 0\}$ is a Zariski-open subset of $\mathfrak{g}_{r}^{*}$. Consequently, $\{f \neq 0\}$ is either empty or else it is dense in $\mathfrak{g}_{r}^{*}$. We have therefore proven:

Lemma 3.2. Let $\mathfrak{g}$ be a 2-step nilpotent Lie algebra. Then $\mathfrak{g}$ is nonintegrable iff there exists two generic points $p, p^{\prime} \in \mathfrak{g}_{r}^{*}$ such that $\operatorname{dim}\left[\mathfrak{g}_{p}, \mathfrak{g}_{p^{\prime}}\right]>0$.

3.1. Geodesic equations of motion. Let $\mathfrak{g}$ be a 2 -step nilpotent Lie algebra. Let $A: \mathfrak{z}^{*} \rightarrow s o(\mathfrak{h})$ be defined for all $x, x^{\prime} \in \mathfrak{h}$ and $q \in \mathfrak{z}^{*}$ by $\left\langle x, A(q) x^{\prime}\right\rangle:=\left\langle q,\left[x, x^{\prime}\right]\right\rangle$. Observe that

Lemma 3.3. $\operatorname{ker} A(q)=\mathfrak{h} \cap \mathfrak{g}_{q}$.

Proof. $x \in \operatorname{ker} A(q) \subset \mathfrak{h}$ iff $0=\left\langle q,\left[x, x^{\prime}\right]\right\rangle=-\left\langle\operatorname{ad}_{x}^{*} q, x^{\prime}\right\rangle$ for all $x^{\prime} \in \mathfrak{h}$. Since $\mathfrak{g}=\mathfrak{h} \oplus Z(\mathfrak{g})$, this is true iff $0=\left\langle q,\left[x, x^{\prime}\right]\right\rangle=-\left\langle\operatorname{ad}_{x}^{*} q, x^{\prime}\right\rangle$ for all $x^{\prime} \in \mathfrak{g}$.

Lemma 3.4. $\mathfrak{g}$ is nonintegrable iff there exists generic points $q, q^{\prime} \in \mathfrak{z}^{*}$ such that $\left[\operatorname{ker} A(q), \operatorname{ker} A\left(q^{\prime}\right)\right] \neq 0$.

Proof. Apply Lemmas 3.2 and 3.3.

Let $(x, y, p, q)$ be the coordinates of a point in $T^{*} G=\mathfrak{h} \times \mathfrak{z} \times \mathfrak{h}^{*} \times \mathfrak{z}^{*}$. The Hamiltonian of the metric $\mathbf{g}$ on $T^{*} G$ is $H_{\mathbf{g}}=\frac{1}{2}\langle p, R p\rangle+\frac{1}{2}\langle q, S q\rangle$ where $R=\mathcal{A}^{-1}$ and $S=\mathcal{B}^{-1}$. The equations of motion are

$$
X_{H_{\mathrm{g}}}= \begin{cases}\dot{q}=0, & \dot{y}=S q+\frac{1}{2}[x, R p] \\ \dot{p}=-A(q) R p, & \dot{x}=R p\end{cases}
$$


Then $q$ is a $\mathfrak{z}^{*}$-valued first integral of $X_{H_{\mathfrak{g}}}$ and $F:=p+A(q) x$ is an $\mathfrak{h}^{*}$-valued first integral. One way to think about the integrability of $X_{H_{\mathbf{g}}}$ is to determine in which cases $F$ can be "pushed down" to $T^{*}(D \backslash G)$.

Let $r^{2}=R$ be a positive-definite, symmetric square root of $R$ (recall that $\mathfrak{h}^{*}$ is identified with $\mathfrak{h}$ via the inner product $\langle\rangle$,$) . Let B(q)=r A(q) r \in s o(\mathfrak{h})$ and $p=r v$, which implies $\dot{v}=-B(q) v$. Because $B(q) \in \operatorname{so}(\mathfrak{h})$, both $\operatorname{ker} B(q)$ and its $\langle$,$\rangle -orthogonal complement are B(q)$ invariant. Let $K_{q}=\operatorname{ker} B(q)$ and $L_{q}=K_{q}^{\perp}$. Then for each $q \in \mathfrak{z}^{*}, \mathfrak{h}=K_{q} \oplus L_{q}$. Note that $K_{q}=r^{-1} \operatorname{ker} A(q)$.

Let $k=\inf _{q \in \mathfrak{z}^{*}} \operatorname{dim} K_{q}$ and $l=\sup _{q \in \mathfrak{z}^{*}} \operatorname{dim} L_{q}$, let $G_{s}\left(\mathfrak{h}^{*}\right)$ denote the Grassmannian of $s$-dimensional planes in $\mathfrak{h}^{*}$ and let $G_{s}=G_{s}\left(\mathfrak{h}^{*}\right) \times \mathfrak{z}^{*}$ denote the trivial bundle. The following is obvious: there is a subset $\mathfrak{z}_{r}^{*} \subset \mathfrak{z}^{*}$ such that $\mathfrak{z}^{*}-\mathfrak{z}_{r}^{*}$ is a closed algebraic set and the map $q \rightarrow K_{q}$ (resp. $q \rightarrow L_{q}$ ) is an algebraic section of $G_{k} \mid \mathfrak{z}_{r}^{*}\left(\operatorname{resp} . G_{l} \mid \mathfrak{z}_{r}^{*}\right)$.

For each $v \in \mathfrak{h}^{*}$ write $v=v_{0}+v_{1}$ where $v_{0}=v_{0}(q) \in K_{q}$ and $v_{1}=v_{1}(q) \in L_{q}$. Abuse notation, and let $B(q)^{-1}=\left(B(q) \mid L_{q}\right)^{-1}$ when $K_{q} \neq \mathfrak{h}^{*}$. We can integrate the equations 3.2 to obtain

$\phi_{t}(x, y, v, q)=\left\{\begin{array}{lll}q(t)=q, & & y(t)=y+t S q+\frac{1}{2} \int_{0}^{t}[x(s), r v(s)] d s, \\ v(t)=e^{-t B(q)} v, & x(t)=x+\operatorname{tr} v_{0}+r B(q)^{-1}\left\{1-e^{-t B(q)}\right\} v_{1} .\end{array}\right.$

Note that $x(t)=t r v_{0}+O(1)=t u+O(1)$, where $u=r v_{0} \in \operatorname{ker} A(q)$. The expression for $y(t)$ may be expanded to yield:

$$
\begin{aligned}
y(t)= & y+t S q+\frac{1}{2} \int_{0}^{t}\left\{\left[x, r v_{0}\right]+\left[x, r e^{-B(q) s} v_{1}\right]\right\} d s \\
& +\int_{0}^{t}\left\{s\left[r v_{0}, r e^{-s B(q)} v_{1}\right]+\left[r B(q)^{-1}\left(1-e^{-s B(q)}\right) v_{1}, r v_{0}+r e^{-s B(q)} v_{1}\right]\right\} d s .
\end{aligned}
$$

Inspection of the integrands shows that the first, second and fourth integrands are bounded functions of $s$. So their integrals have a norm bounded by const. $\times t$. The substitution $u=s r v_{0}$ and $d v=r e^{-B(q) s} v_{1} d s$ combined with integration by parts on the third integrand shows that its norm is bounded by const. $\times t$, also.

Lemma 3.5. Let $P=(x, y, p, q) \in T^{*} G$ be such that $B(q) \neq 0$. Let $p=r v$ and write $v=v_{0}+v_{1}$ (resp. $p=p_{0}+p_{1}$ ) with $p_{i}=r v_{i}$. Assume that $v_{0} \neq 0$. Then the projective asymptotic homotopy class of $\phi_{t}(P)$ is nontrivial and $[P]_{2, \phi} \subseteq \hat{\pi}\left(p_{0}+\mathfrak{z}\right)$.

Proof. Let $\mathbf{p}: T^{*} G \rightarrow G$ denote the canonical projection. Assume that $(x, y)=$ $(0,0)$. Since $|y(t)| \leq$ const. $\times t, v_{0} \neq 0, x(t)=t r v_{0}+O(1)$ and $p_{0}=r v_{0}$, we have that $\mathbf{p} \phi_{t}(P) /\left|\mathbf{p} \phi_{t}(P)\right|=c(t) p_{0}+z(t)$, where $c(t)$ is a positive function of $t$ bounded away from 0 and $\infty$ and $z(t) \in \mathfrak{z}$ has norm $<1$. Thus $\pi\left(\mathbf{p} \phi_{t}(P)\right)=\pi\left(p_{0}+c(t)^{-1} z(t)\right)$. Since $\left|c(t)^{-1} z(t)\right| \leq$ const., this shows that $[P]_{2, \phi} \subseteq \hat{\pi}\left(p_{0}+\mathfrak{z}\right)$.

Assume now that $g=(x, y) \neq(0,0)$. The left-invariance of $\phi_{t}$ and the results of the previous paragraph show that $[P]_{2, \phi} \subseteq \hat{\pi}\left(\operatorname{Ad}_{g} p_{0}+\mathfrak{z}\right)$. Since $\mathfrak{g}$ is 2-step nilpotent, one computes that $\operatorname{Ad}_{g} p_{0}+\mathfrak{z}=p_{0}+\mathfrak{z}$, which proves the lemma.

Corollary 3.6. Assume the hypotheses of the previous lemma. Then the free projective asymptotic homotopy class of $\phi_{t}(P)$ is nontrivial and $\mathcal{F}_{2, \phi}(P) \subseteq \hat{\pi}\left(p_{0}+\mathfrak{z}\right)$.

Proof. Apply the previous lemma and the definition of $\mathcal{F}$. 
Proposition 3.7. Assume that $\mathfrak{g}$ is a nonintegrable 2-step nilpotent Lie algebra with a $\mathbf{Q}$-structure. Let $D<G$ be a discrete cocompact subgroup, $\Sigma=D \backslash G$ and $\mathrm{g}$ be a left-invariant metric on $G$. Then the geodesic flow of $\mathbf{g}$ on $T^{*} \Sigma$ is not integrable.

Proof. Let $\hat{\phi}_{t}$ (resp. $\left.\phi_{t}\right)$ denote the geodesic flow of $\mathbf{g}$ on $T^{*} \Sigma\left(\right.$ resp. $\left.T^{*} G\right)$. The geodesic flows satisfy $\hat{\phi}_{t}(D P)=D \phi_{t}(P)$ for all $P \in T^{*} G$. Let $D P, D P^{\prime} \in T^{*} \Sigma$. From the definition of $\mathcal{F}, \mathcal{F}_{n, \hat{\phi}}(D P)=\mathcal{F}_{n, \phi}(P)$ and similarly for $D P^{\prime}$. By Corollary 3.6, $\{\overline{0}\} \neq \mathcal{F}_{2, \phi}(P) \subseteq \hat{\pi}\left(p_{0}(q)+\mathfrak{z}\right)$ and $\{\overline{0}\} \neq \mathcal{F}_{2, \phi}\left(P^{\prime}\right) \subseteq \hat{\pi}\left(p_{0}^{\prime}\left(q^{\prime}\right)+\mathfrak{z}\right)$. Let us remark that if $\bar{x} \in \mathcal{F}_{2, \phi}(P)$ and $\bar{x}^{\prime} \in \mathcal{F}_{2, \phi}\left(P^{\prime}\right)$ are both nontrivial elements, then $\left[\bar{x}, \bar{x}^{\prime}\right]=\pi\left(\left[p_{0}(q), p_{0}^{\prime}\left(q^{\prime}\right)\right]\right)$. By Lemma 3.4 and the subsequent discussion, $p_{0}(q) \in \mathfrak{g}_{q} \cap \mathfrak{h}$ and $p_{0}^{\prime}\left(q^{\prime}\right) \in \mathfrak{g}_{q^{\prime}} \cap \mathfrak{h}$. By the hypothesis that $\mathfrak{g}$ is nonintegrable and 2 -step nilpotent, it follows that for an open dense set of $\left(q, q^{\prime}\right) \in \mathfrak{z}^{*} \times \mathfrak{z}^{*}$ there exists $u_{0} \in \mathfrak{g}_{q} \cap \mathfrak{h}, u_{0}^{\prime} \in \mathfrak{g}_{q^{\prime}} \cap \mathfrak{h}$ such that $\left[u_{0}, u_{0}^{\prime}\right] \neq 0$. Thus, for an open and dense set of $\left(D P, D P^{\prime}\right) \in T^{*} \Sigma \times T^{*} \Sigma$ the free projective asymptotic homotopy classes $\mathcal{F}_{2, \hat{\phi}}(D P)$ and $\mathcal{F}_{2, \hat{\phi}}\left(D P^{\prime}\right)$ are nontrivial and do not weakly commute.

Assume $\hat{\phi}_{t}: T^{*} \Sigma \rightarrow T^{*} \Sigma$ is integrable in the sense of Definition 1.2 Then there exists an open dense set $L \subset T^{*} \Sigma$ such that $L=\bigcup L_{\alpha}$ and the open sets $L_{\alpha}$ are $\hat{\phi}_{t}$-invariant and have an abelian fundamental group. Thus, for an open dense set of points $D P \in T^{*} \Sigma$ there is an open neighbourhood of $D P, L_{\alpha(D P)} \subset$ $L$, which is $\hat{\phi}_{t}$-invariant and has an abelian fundamental group. Since the free projective asymptotic homotopy classes of an open dense set of points $\left(D P, D P^{\prime}\right) \in$ $L_{\alpha} \times L_{\alpha}$ are nontrivial, Lemma 2.7] implies that $\mathcal{F}_{2, \hat{\phi}}(D P)$ and $\mathcal{F}_{2, \hat{\phi}}\left(D P^{\prime}\right)$ weakly commute. Therefore, there is an open set of points $\left(D P, D P^{\prime}\right) \in T^{*} \Sigma \times T^{*} \Sigma$ with weakly commuting free projective asymptotic homotopy classes. This contradicts the conclusion of the previous paragraph, which is absurd.

\section{REFERENCES}

1. H. Bass, The degree of polynomial growth of finitely generated nilpotent groups, Proc. London Math. Soc. (3) 25, 603-614 (1972). MR 52:577

2. D. Benardete and J. Mitchell, Asymptotic homotopy cycles for flows and $\Pi_{1}$ de Rham theory, Trans. Amer. Math. Soc. 338(2), 495-535 (1993). MR 93j:58107

3. A. V. Bolsinov and I. A. Taimanov, Integrable geodesic flows with positive topological entropy, Invent. Math. 140(3), 639-650 (2000). MR 2001b:37081

4. L. T. Butler, A new class of homogeneous manifolds with Liouville-integrable geodesic flows, C. R. Math. Acad. Sci. Soc. R. Can. 21(4), 127-131 (1999). MR 2001i:53141

5. L. T. Butler, New examples of integrable geodesic flows, Asian J. Math. 4(3), 515-526 (2000). MR 2001i:37090

6. L. T. Butler, Integrable geodesic flows with wild first integrals: The case of two-step nilmanifolds, Ergodic Theory Dynam. Systems, to appear.

7. L. T. Butler, Integrable geodesic flows on n-step nilmanifolds, J. Geom. Phys. 36(3-4), 315-323 (2000). MR 2002j:37077

8. L. T. Butler, Invariant metrics on a nilmanifold with positive topological entropy, submitted to Geometriae Dedicata. 2001.

9. K. T. Chen, Extension of $C^{\infty}$ function algebra by integrals and Malcev completion of $\pi_{1}$. Advances in Math. 23(2), 181-210 (1977). MR 56:16664

10. P. Eberlein, Geometry of 2-step nilpotent groups with a left invariant metric, Ann. Sci. École Norm. Sup. 27(5), 611-660 (1994). MR 95m:53059

11. P. Eberlein, Geometry of 2-step nilpotent groups with a left invariant metric. II, Trans. Amer. Math. Soc. 343(2), 805-828 (1994). MR 95b:53061

12. F. Fried, The geometry of cross sections to flows, Topology 21(4), 353-371 (1982). MR 84d:58068 
13. C. S. Gordon and E. N. Wilson, The spectrum of the Laplacian on Riemannian Heisenberg manifolds, Michigan Math. J. 33(2), 253-271 (1986). MR 87k:58275

14. A. B. Katok, Ergodic perturbations of degenerate integrable Hamiltonian systems, Izv. Akad. Nauk SSSR Ser. Mat. 37, 539-576 (1973). MR 48:9758

15. K. B. Lee and K. Park, Smoothly closed geodesics in 2-step nilmanifolds, Indiana Univ. Math. J. 45(1), 1-14 (1996). MR 97h:53044

16. A. I. Malcev, On a class of homogeneous spaces, Amer. Math. Soc. Translation, no. 39, 1951. MR 12:589e

17. M. Mast, Closed geodesics in 2-step nilmanifolds, Indiana Univ. Math. J. 43, 885-911 (1994). MR 96a:53057

18. G. P. Paternain, Geodesic flows, Progress in Math., vol. 180, Birkhäuser, Boston, MA, 1999. MR 2000h:53108

19. F. Rhodes, Asymptotic cycles for continuous curves on geodesic spaces, J. London Math. Soc. (2), 6, 247-255 (1973). MR 49:6208

20. S. Schwartzman, Asymptotic cycles, Ann. of Math. (2) 66, 270-284 (1957). MR 19:568i

Department of Mathematics, Northwestern University, 2033 Sheridan Road, EVANSTON, ILLINOIS 60208

E-mail address: lbutler@math.northwestern.edu 